\title{
RELACIÓN ENTRE LA FLEXIBILIDAD DE LOS ISQUIOTIBIALES Y LA FUERZA DEL SOLEO CON EL SALTO Y LA VELOCIDAD EN JUGADORES DE ULTIMATE FRISBEE
}

\author{
RELATIONSHIP BETWEEN THE FLEXIBILITY \\ OF THE HAMSTRING AND THE STRENGTH \\ OF SOLEUS MUSCLE WITH JUMP AND SPEED \\ IN ULTIMATE FRISBEE PLAYERS
}

\section{RELAÇÃO ENTRE A FLEXIBILIDADE DOS ISQUIOTIBIAIS, A FORÇA DO SÓLEO COM O SALTO E A VELOCIDADE EMJOGADORES DE ULTIMATE FRISBEE}

\section{German Alberto Rincón Rey ${ }^{1}$ Santiago Cadavid Escobar ${ }^{2}$}

1 Magister en Actividad F sica y Deporte. Universidad Aut noma de Manizales. Colombia. ORCID: https://orcid.org/0000-0001-5149-8820 E-mail: german. rinconr@autonoma.edu.co

2 Magister en Actividad F sica y Deporte. Universidad Aut noma de Manizales. Colombia. ORCID: https://orcid.org/0000-0003-1363-1664

Rinc n Rey, G. A. \& Cadavid Escobar, S. (2017). Relaci n entre la flexibilidad de los isquiotibiales y la fuerza del soleo con el salto y la velocidad en jugadores de ultimate frisbee. Educaci n F sica y Deporte, 36(2), 244-263. Jul.-Dic. http://doi. org/10.17533/udea.efyd.v36n2a05

10.17533/udea.efyd.v36n2a05

URL DOI: http://doi.org/10.17533/udea.efyd.v36n2a05

Art culo de investigaci $n$ 


\section{RESUMEN}

Problema: la reducci $\mathrm{n}$ de la flexibilidad de los isquiotibiales es com $\mathrm{n}$ en adolescentes, asoci ndose con dolor lumbar, hernia de disco y riesgo de lesiones musculares. El ultimate frisbee implica aceleraciones y desaceleraciones en cortas distancias. Los antecedentes reportan lesiones en miembros inferiores, sin correlacionarlas con patrones de movimiento como fuerza, velocidad o salto. Objetivo: determinar la relaci $n$ entre la flexibilidad de los isquiotibiales y la fuerza del soleo, con el salto y la velocidad en jugadores de ultimate frisbee. $\mathbf{M}$ todo: estudio descriptivo, correlacional, con 103 deportistas universitarios de Medell n, Colombia. La informaci $n$ se recolect mediante encuesta y observaci $n$. Se evalu la flexibilidad, la velocidad y el salto. Los datos se analizaron con SPSS, versi n 24.0. Resultados: la flexibilidad de los isquiotibiales se correlacion positivamente con la velocidad, pero inversamente con el salto. Conclusi $\mathbf{n}$ : a mayor flexibilidad de los isquiotibiales, mayor desarrollo de velocidad, pero menor capacidad de salto.

PALABRAS CLAVE: salto contragravedad, test de Wells, fuerza $m$ xima, desaceleraci $n$, ultimate frisbee, deportistas universitarios.

\section{ABSTRACT}

Problem: Reduction of hamstring flexibility is common in adolescents, associating with low back pain, disc herniation, and risk of muscle injuries. Ultimate frisbee implies accelerations and decelerations over short distances. The background reports injuries in the lower limbs, without correlating them with movement patterns such as strength, speed or jump. Objective: To determine the relationship between hamstring flexibility and the strength of the soleus muscle with jump and speed in ultimate frisbee players. Method: Descriptive and correlational study, with 103 university athletes from Medellin, Colombia. Information was collected through survey and observation. Flexibility, speed and jump were evaluated. Data were analyzed with SPSS ${ }^{\circledR}$ version 24.0. Results: Hamstring flexibility was positively correlated with speed, but inversely with jump. Conclusion: A greater flexibility of the hamstrings, greater speed development, but less jumping ability.

KEYWORDS: Antigravity jump, Wells test, maximum strength, deceleration, ultimate frisbee, university athletes. 


\section{RESUMO}

Problema: a reduç o da flexibilidade dos isquiotibiais um quadro $\mathrm{Cl}$ nico comum emadolescentes, esta reduç o foi reportada como um fator associado preval ncia de dor lombar, $\mathrm{h}$ rnia de disco e alto risco de les es musculares. O ultimatefrisbee combina padr es de movimento similares ao futebol e ao handebol, onde se realizam cortes, arremessos, saltos, velocidade, voos e aceleraç o -desaceleraç o em curtas dist ncias. Os antecedentes encontrados nesse esporte reportam ndices de les es em membros inferiores, mas $\mathrm{n}$ o se constataramcorrelaç es entre padr es de movimento espec fico com o salto, a força e a velocidade. Objetivo: determinar a relaç o da flexibilidade dos isquiotibiais, a força do s leo com o salto e avelocidade em jogadores de ultimate frisbee. M todo: realizou-se um estudo descritivo com alcance correlacional e amostra de conveni ncia de 103 jogadoresuniversit rios de ultimate frisbee da cidade de Medellin-Col mbia. Os esportistas cumpriram os crit rios de inclus o e assinaram o consentimentoinformado. No que se refere $\mathrm{s}$ t cnicas de coleta de informaç o, utilizou-se a pesquisa de opini o e a observaç o. Avaliou-se a flexibilidade, a velocidade eo salto. Os dados se analisaram com o programa estad stico SPSS vers o 24.0. Realizou-se uma an lise uni-variada de estad stica descritiva, que confirmou asfrequ ncias absolutas e relativas. Al m disso, determinouse a distribuiç o da normalidade de todas as vari veis atrav $\mathrm{s}$ do teste Kolmogorov-Smirnov, queassinalou normalidade para as vari veis com um valor < 0,05. Resultados: estabeleceu-se que a flexibilidade dos isquiotibiais tem correlaç o positivacom a velocidade, mas inversa com o salto. Conclus o: quanto maior a flexibilidade dos isquiotibiais, maior o desenvolvimento da velocidade, por mmenor a capacidade de salto.

PALAVRAS CHAVES: salto contra gravitacional, teste de Wells, força m xima, desaceleraç o, ultimatefrisbee, esportistas universit rios. 


\section{INTRODUCCIÓN}

\section{Ultimate Frisbee}

El ultimate frisbee es un deporte mixto de no contacto, autorregulado, jugado con un disco volador, que combina patrones de otros deportes como futbol, balonmano, baloncesto y futbol americano. La din mica de juego consiste en el encuentro de dos equipos, cada uno conformado por 7 jugadores, en un campo juego de $65 \times 36 \mathrm{mts}$, con zonas de anotaci $\mathrm{n}$ de 23 metros, durante 100 minutos o 15 anotaciones, seg $\mathrm{n}$ lo establece la Federaci n Internacional de Disco Volador (WFDF). Un aspecto relevante de este deporte, que lo hace diferente a otros, es el "esp ritu de juego", donde cada jugador debe comprometerse a respetar las reglas y no infringirlas voluntariamente (Smith, Greenlees \& Manley, 2009, p.746). En este sentido, la figura de rbitro no es utilizada en el Ultimate, ya que cada jugador, honestamente, debe estar pendiente de sus infracciones y de las de los rivales. Seg $\mathrm{n}$ la WFDF, se busca la $\mathrm{m}$ xima competitividad, pero nunca si eso implica perder el respeto entre los jugadores, el cumplimiento de las reglas y el placer de jugar (Smith et al., 2009, p.746).

\section{Flexibilidad de los Isquiotibiales}

La flexibilidad es la capacidad $\mathrm{f}$ sica de amplitud de movimientos en una, o en un grupo de articulaciones. Algunos autores plantean que la flexibilidad abarca otros aspectos adem s de los movimientos articulares, como la capacidad el stica muscular y las deformaciones de los componentes articulares (Arregui \& Mart nez, 2001, p.127). Los $m$ sculos que com nmente presen$\tan \mathrm{m} \mathrm{s}$ ndices de lesiones en el deporte son los isquiotibiales, el recto femoral y la cabeza medial de los gastrognemios (Valle et al., 2015, p.11). El semimembranoso y semitendinoso, y la cabeza larga del b ceps femoral, son biarticulares, en tanto que la cabeza corta del b ceps femoral es monoarticular (Valle et al., 2015, p.11). 
Los isquiotibiales atraviesan dos articulaciones, lo que permite, simult neamente, la flexi $\mathrm{n}$ de rodilla y la extensi $\mathrm{n}$ de cadera. Esta anatom a biarticular algunas veces significa que los $\mathrm{m}$ sculos isquiotibiales tienen una pesada carga sobre dos articulaciones simult neamente, como una contracci n exc ntrica en la cadera y en la rodilla en la fase final de balanceo en ciclo de la marcha (Chumanov, Heiderscheit \& Thelen, 2011, p.527). Otros autores definen la flexibilidad como una propiedad intr nseca de los tejidos del cuerpo, que determina el rango de movimiento articular realizado sin lesi $\mathrm{n}$ en una, o en un grupo de articulaciones (Bandy, Irion \& Briggler, 1997, p.5). Esta propiedad del sistema m sculo esquel tico, puede ser examinado por dos clases de medidas biomec nicas: flexibilidad est tica y flexibilidad din mica.

La flexibilidad est tica "es una medida cl nica que define la cantidad de movimiento de una, o de un grupo de articulaciones" (Rogan, W st, Schwitter \& Schmidtbleicher, 2015, p.4). La flexibilidad din mica es el incremento de la resistencia con la elongaci $n$ muscular, dado por el rango de movimiento articular, que puede ser cuantificado en t rminos de rigidez (Chumanov et al., 2011, p.527; Rogan et al., 2015, p.4). La flexibilidad pasiva y activa se puede medir mediante la rigidez muscular o los grados de movimiento articular disponibles en una, o en un grupo de articulaciones, debido a la elongaci $\mathrm{n}$ de las fibras musculares y el tejido conectivo (Askling, Saartok \& Thorstensson, 2006, p.42).

La disminuci $\mathrm{n}$ de la flexibilidad en los miembros inferiores puede resultar en cambios en la din mica del rango de movimiento articular, en la biomec nica, en el rendimiento y en el riesgo de lesiones (Hammonds et al., 2012, p.6). Una reducci $n$ en la flexibilidad de los isquiotibiales, ha sido reportada como un factor de asociaci $\mathrm{n}$ con la ocurrencia de dolor lumbar en adolescentes y adultos (Nishikawa et al., 2015, p.3). Una reducci $n$ en la flexibilidad de los $m$ sculos isquiotibiales ha sido reportada como un incremento en el riesgo de da o del sistema m sculo esquel tico (Muehlbauer, 
Gollhofer \& Granacher, 2015, p.1673; Hammonds et al., 2012, p.6; Malliaropoulos et al., 2015, p.204). As, la flexibilidad de los isquiotibiales es importante para la salud en general y para el ejercicio f sico (Daneshjoo, Rahnama, Mokhtar \& Yusof, 2013, p.46; Gabbe, Finch, Bennell, \& Wajswelner, 2005, p.107; Nishikawa et al., 2015, p.3; Czaprowski et al., 2013, p.6).

\section{Fuerza Máxima}

La fuerza $m$ xima se define como la $m$ xima tensi $n$ manifestada por el m sculo o conjunto de grupos musculares, en la que se puede completar un arco de movimiento articular con una carga externa m xima (Gonz lez \& Gorostiaga, 2002, p.120). Algunos autores reportan que porcentajes entre $65-85 \%$ de una repetici $n$ $\mathrm{m}$ xima (1RM) son ptimos para la hipertrofia muscular (Jenkins et al., 2016, p.136). Otros autores plantean que la fuerza m xima depende de un factor determinante como es la velocidad, ya que cuando esta act a durante un patr $\mathrm{n}$ de movimiento, el porcentaje de fuerza disminuye (Gonz lez \& Gorostiaga, 2002, p.120). En deportes como futbol y baloncesto, el entrenamiento de fuerza $\mathrm{m}$ xima mayor a $85 \% 1 \mathrm{RM}$ ha perdido importancia debido a la $\mathrm{p}$ rdida de velocidad de contracci n muscular y a la eficacia del movimiento (Gonz lez \& Gorostiaga, 2002, p.120; Beedie, 2007, p.22; Domire \& Challis, 2015, p.26). En estos deportes, los porcentajes de 1RM oscilan entre $25-30 \%$, pero la fuerza debe estar orientada a la optimizaci $n$ del gesto deportivo (Domire \& Challis, 2015, p.26). Actualmente la fuerza m xima se utiliza en deportes de halterofilia, como el levantamiento de pesas, lanzamiento de martillo y otros.

\section{Salto Vertical}

El salto es una habilidad fundamental en diversos deportes, como baloncesto, voleibol, balonmano o futbol. En el contexto de estos deportes, un salto con un buen desplazamiento del centro de masa, no siempre es un indicador de una buena habilidad o 
ejecuci $n$ del salto (Domire \& Challis, 2015, p.26). En el salto vertical, quiz s sea necesario referirse a dos mecanismos que act an durante este patr $\mathrm{n}$ espec fico de movimiento. El primer mecanismo que act a en el salto es el ciclo acortamientoalargamiento (Asadi \& Ram rez, 2016, p.46; Arazi, Mohammadi \& Asadi, 2014, p.126). Este ciclo consta de una $r$ pida contracci $n$ exc ntrica, seguida de una contracci n conc ntrica, que es la propulsora del despegue (Francis, Lenz, Lenhart \& Thelen 2013, p. 994; Arazi et al., 2014, p.126). El segundo mecanismo se da desde que termina la propulsi $n$, hasta la ca da al suelo, en la que los isquiotibiales y el soleo realizan una contracci n conc ntrica para amortiguar la fuerza del cuerpo en ca da (Gambelli, Theisen, Willems \& Schepens, 2015, p.11; Wong, Bobbert, Soest, Gribble \& Kistemaker, 2016, p.4).

Un reciente meta an lisis, documenta que el aumento de la masa corporal y la aceleraci $\mathrm{n}$ gravitacional, inducida por una resistencia el stica durante la fase exc ntrica del salto, genera un aumento en el ciclo estiramiento-acortamiento durante el salto (Aboodarda, Page \& Behm, 2015, p.841). Esto podr a obedecer a la experiencia activa muscular durante los saltos en la fase exc ntrica, almacenando energ a el stica en los componentes contr ctiles de las estructuras musculo-tendinosas, mejorando la potenciaci $n$ mioel ctrica y los mecanismos reflejos de estiramiento (Aboodarda, Page \& Behm, 2015, p.842).

\section{METODOLOGÍA}

El presente estudio fue de tipo descriptivo, con alcance correlacional y muestreo a conveniencia de 103 sujetos, de un total de 140 jugadores universitarios de ultimate frisbee de la ciudad de Medell n. Todos los deportistas cumplieron con los criterios de inclusi n y firmaron el consentimiento informado, acorde con lo establecido por la Resoluci n 08430 sobre 
investigaci $\mathrm{n}$ en salud como riesgo $\mathrm{m}$ nimo. Como $\mathrm{t}$ cnicas de recolecci $\mathrm{n}$ de informaci $\mathrm{n}$ se utilizaron la encuesta y la observaci n. Los instrumentos empleados fueron el formato de encuesta de las variables sociodemogr ficas y las pruebas de evaluaci $\mathrm{n}$ de la flexibilidad, con el test de Wells; velocidad, con el test de 30 metros; y salto, con el test de salto contra gravedad. Los datos fueron analizados por el programa estad stico SPSS versi n 24.0. Se realiz un an lisis univariado, empleando estad stica descriptiva, que dio raz $\mathrm{n}$ a las frecuencias absolutas y relativas. Se determin la distribuci $n$ de normalidad de todas las variables, a trav s de la prueba Kolmogorov-Smirnov, estableciendo normalidad para las variables con un $\mathrm{P}$ valor $<0,05$.

\section{RESULTADOS}

La muestra de investigaci $\mathrm{n}$ fue de 103 jugadores universitarios de ultimate frisbee de la ciudad de Medell $n, 68$ hombres y 35 mujeres, con media de edad de 21 a os +/- 3,14 a os. En la tabla 1 se caracteriz la flexibilidad de los isquiotibiales seg $n$ el g nero, donde la media de la flexibilidad de las mujeres es aproximadamente $5 \mathrm{cms}$ mayor que en los hombres, siendo esta diferencia estad sticamente significativa.

Tabla 1. Caracterización de la flexibilidad según género (test de Wells: cms).

\begin{tabular}{cccccccc}
\hline Sexo & $N$ & Mínimo & Máximo & Media & $\begin{array}{c}\text { Desviación } \\
\text { estándar }\end{array}$ & $\begin{array}{c}T \\
\text { student }\end{array}$ & Significa. \\
\hline Masculino & 68 & 20 & 59 & 34,71 & 8,816 & $-2,63$ & 0,010 \\
Femenino & 35 & 26 & 61 & 39,29 & 7,370 & & \\
\hline
\end{tabular}

La tabla 2 muestra la caracterizaci $\mathrm{n}$ de la fuerza $\mathrm{m}$ xima del soleo seg $\mathrm{n}$ el $\mathrm{g}$ nero, donde la media de la fuerza $\mathrm{m}$ xima del soleo entre el pie izquierdo y el derecho fueron similares 
para ambos g neros. Sin embargo, la fuerza $m$ xima del soleo de los hombres fue aproximadamente 30 libras mayor que la de las mujeres.

Tabla 2. Caracterización de la fuerza máxima de soleo según género.

\begin{tabular}{cllcccc}
\hline \multirow{2}{*}{ Sexo } & Fuerza soleo & $N$ & Mínimo & Máximo & Media & $\begin{array}{c}\text { Desviación } \\
\text { estándar }\end{array}$ \\
\hline \multirow{2}{*}{ Masculino } & Pie izquierdo & 68 & 40 & 290 & 155,76 & 66,433 \\
& Pie derecho & 68 & 40 & 340 & 158,41 & 71,471 \\
\multirow{2}{*}{ Femenino } & Pie izquierdo & 35 & 10 & 290 & 124,03 & 79,300 \\
& Pie derecho & 35 & 10 & 310 & 123,31 & 79,994 \\
\hline
\end{tabular}

En la tabla 3 se caracterizaron las variables de estudio salto y velocidad seg $\mathrm{n}$ el g nero, reflejando que la media de la velocidad y el salto es mejor en los hombres que en las mujeres.

Tabla 3. Caracterización del salto y la velocidad según género.

\begin{tabular}{cllcccc}
\hline \multirow{2}{*}{ Sexo } & \multicolumn{1}{c}{ Variable } & $N$ & Mínimo & Máximo & Media & $\begin{array}{c}\text { Desviación } \\
\text { estándar }\end{array}$ \\
\hline \multirow{2}{*}{ Masculino } & Velocidad en segundos & 68 & 3,01 & 5,14 & 3,75 &, 391 \\
& Altura en centímetros & 68 & 23,8 & 52,5 & 39,8 & 6,028 \\
& Velocidad en segundos & 35 & 3,83 & 6,30 & 4,7 &, 520 \\
\multirow{2}{*}{ Femenino } & Altura en centímetros & 35 & 15,4 & 35,2 & 26,4 & 5,001 \\
\hline
\end{tabular}

En el an lisis Bivariado, se realiz correlaci $\mathrm{n}$ entre las variables de estudio y algunas variables sociodemogr ficas y antropo$\mathrm{m}$ tricas que se muestran en la tabla 4, encontrando que existe significancia estad stica y correlaci $n$ baja negativa entre la flexibilidad de los isquiotibiales con respecto a la edad, la talla y el peso, lo que supone que los jugadores con menor edad, menor altura y menor peso, tienen mejor flexibilidad de isquiotibiales.

En la tabla 5 se muestra la correlaci $\mathrm{n}$ de las variables de estudio como la flexibilidad de los isquiotibiales, con la fuerza del 
soleo, el salto y la velocidad, encontrando que la flexibilidad de los isquiotibiales tiene una correlaci $\mathrm{n}$ directa con la velocidad, e inversa con el salto; en cambio, la fuerza $\mathrm{m}$ xima del soleo tiene una relaci $\mathrm{n}$ inversa con la velocidad y directa con el salto.

Tabla 4. Correlación de la flexibilidad con la edad, peso y talla.

\begin{tabular}{llcccc}
\hline & $\begin{array}{c}\text { Wells en } \\
\text { centímetros }\end{array}$ & $\begin{array}{c}\text { Edad en } \\
\text { años }\end{array}$ & $\begin{array}{c}\text { Peso en } \\
\text { kilogramos }\end{array}$ & $\begin{array}{c}\text { Talla en } \\
\text { metros }\end{array}$ \\
\hline $\begin{array}{l}\text { Wells en } \\
\text { centímetros }\end{array}$ & $\begin{array}{l}\text { Correlación de } \\
\text { Spearman }\end{array}$ & 1 &,$- 284^{\star \star}$ &,$- 251^{*}$ &,- 210 \\
& Sig. (bilateral) & &, 004 &, 011 &, 034 \\
& $\mathrm{~N}$ & 103 & 103 & 103 & 103 \\
\hline
\end{tabular}

$\mathrm{N} * *$ La correlación es significativa en el nivel 0,01 (bilateral). * La correlación es significativa en el nivel 0,05 (bilateral).

\section{DISCUSIÓN}

Para efectos de la presente investigaci $n$, la muestra de estudio correspondi a 103 jugadores universitarios de Ultimate frisbee de la ciudad de Medell $n$. Se evidenci que la mayor poblaci $n$ evaluada pertenece al g nero masculino, de los cuales 68 fueron hombre y 35 mujeres. Estos resultados se asemejan a estudios realizados en Medell n (Tejada \& Ram n, 2013, p.4), donde la poblaci $\mathrm{n}$ fueron 13 hombres y 11 mujeres jugadores de la selecci $n$ Colombia de Ultimate, al igual que otro estudio realizado en Estados Unidos (Reynolds \& Halsmer, 2006, p.47), donde fueron 79 hombres y 56 mujeres. Aunque dista de lo encontrado por (Ram rez et al., 2017, p.995) donde, de una poblaci n de 7614 estudiantes colombianos de los cuales 3258 fueron hombres y 4356 mujeres.

En el presente estudio se encontr que las mujeres tuvieron mayor flexibilidad de isquiotibiales que los hombres, con una media de flexibilidad de $39,69 \mathrm{cms}$, resultados similares a 
Tabla 5. Correlación entre la flexibilidad y fuerza del soleo con el salto y la velocidad

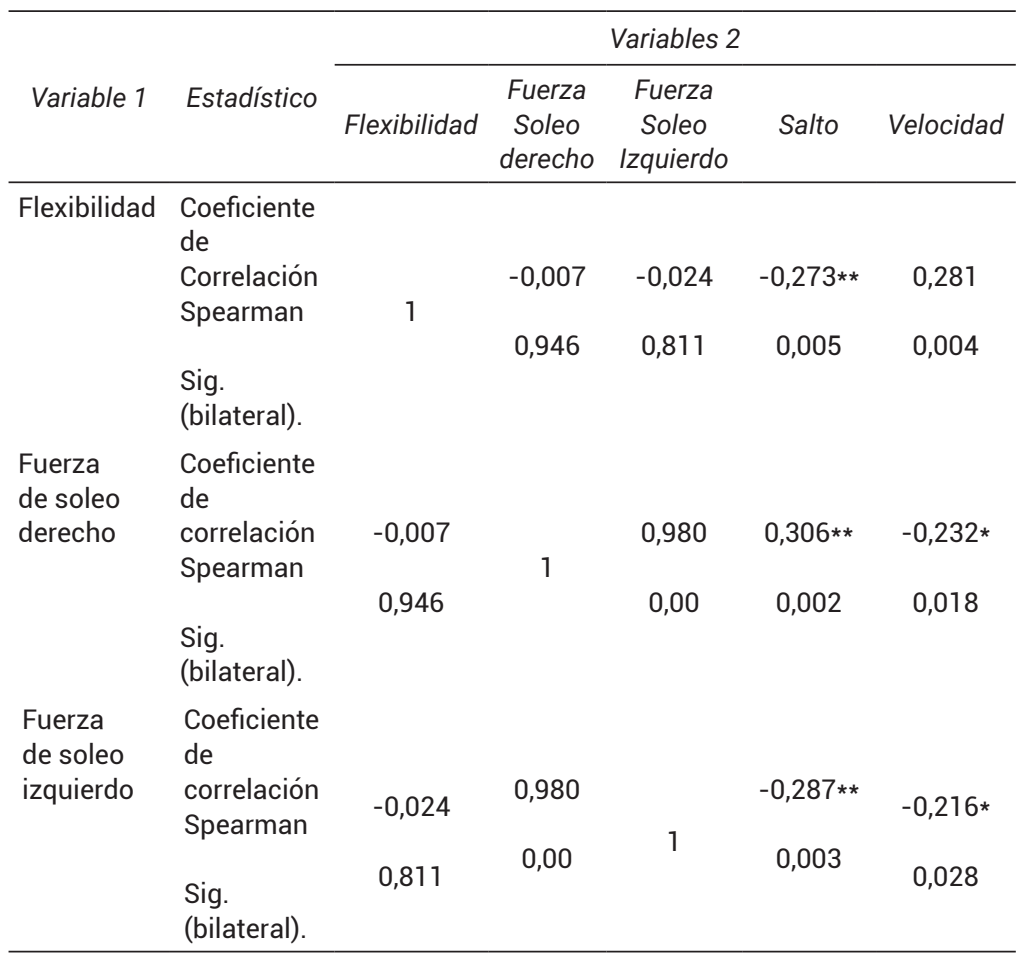

Nota: ** La correlación es significativa en el nivel 0,01 (bilateral). * La correlación es significativa en el nivel 0,05 (bilateral).

los reportados por Malliaropoulos et al. (2015, p.204), donde las mujeres corredoras tuvieron mayor rango de movilidad articular de rodilla, con una media de $73,2^{\circ}$. Tambi $\mathrm{n}$ cabe resaltar que esto puede estar asociado a factores de riesgo de lesiones, ya que, de acuerdo con lo reportado por Akinbola, Logerstedt, Hunter \& Snyder (2015, p.76), las mujeres tuvieron un ndice de lesi $\mathrm{n}$ en miembros inferiores de $70.6 \%$, en comparaci $\mathrm{n}$ con los hombres, con $29.4 \%$. Siendo el ultimate un deporte de no contacto, estos ndices de lesiones pueden estar asociados a 
fuerza muscular, inestabilidad articular y adaptaci $\mathrm{n}$ a la pr ctica deportiva (Reynolds \& Halsmer, 2006, p.48). Lo anterior difiere de lo encontrado por Hammonds et al. (2012, p.7), donde no hubo diferencias significativas en la flexibilidad de isquiotibiales en hombres y mujeres despu s de un protocolo de estiramientos. Otros estudios se alan que las diferencias en la flexibilidad entre hombres y mujeres podr an estar asociadas a otras variables como tipo de deporte, desarrollo de la fuerza, tiempo y frecuencia de entrenamiento, $\mathrm{m}$ s que a condiciones fisiol gicas de cada g nero (Hammonds et al., 2012, p.10; Muehlbauer et al., 2015, p.1684).

En el presente estudio, las capacidades f sicas como salto, velocidad y fuerza, fueron mayores en hombres que en mujeres, asemej ndose a lo hallado en otros estudios (Jenkins et al., 2016, p.138; Sabido, Hern ndez, Botella \& Moya, 2016, p.8; Tejada \& Ram n, 2013, p.5). Estas caracter sticas podr an estar asociadas a la anatom a y fisiolog a masculina, en las cuales la fuerza tiene un mayor desarrollo debido a componentes hormonales como la testosterona y el volumen de la masa muscular (Arazi et al., 2014, p.126; Wong et al., 2016, p.10). Por otra parte, los hombres tuvieron una media de $39,8 \mathrm{~cm}$ de altura en el test de salto, y $3,75 \mathrm{seg}$ en el test de velocidad de $30 \mathrm{mts}$, mientras las mujeres alcanzaron $26,4 \mathrm{~cm}$ de altura y $4,7 \mathrm{seg}$ en velocidad, similar a lo reportado por Tejada \& Ram n (2013, p.6), donde los hombres presentaron una media $54,3 \mathrm{~cm}$ y $4,5 \mathrm{seg}$, y las mujeres $40,7 \mathrm{~cm}$ y 5,1 seg respectivamente. Estos datos tambi $\mathrm{n}$ se asemejan a los hallazgos de Ram rez et al. (2017, p.995), donde hombres j venes en edades entre 7 y 18 a os alcanzaron un $\mathrm{m}$ ximo de $38 \mathrm{~cm}$, y mujeres $27 \mathrm{~cm}$ en el test de salto vertical. Los datos del presente estudio se encuentran en el percentil 1, tanto en hombres como en mujeres, seg $\mathrm{n}$ la tabla de altura de salto establecida por Huerta, Chirosa, Guisado \& C ceres (2016, p.753). Para el test de velocidad de 30mts, los hombres tuvieron una calificaci $\mathrm{n}$ excelente y las mujeres una calificaci n promedio, 
seg $\mathrm{n}$ la escala de valoraci $\mathrm{n}$ del tiempo en segundos (Rodr guez \& And jar, 2010, p.6).

Esta diferencia entre los grupos podr a deberse a que los sujetos evaluados por Tejada \& Ram n (2013, p.6) fueron deportistas de alto rendimiento, con una frecuencia alta de entrenamiento, quienes se preparaban para un torneo internacional, en comparaci $\mathrm{n}$ con los jugadores universitarios amateurs del presente estudio. Al igual, las tablas de referencia para los test de salto y velocidad fueron establecidas para deportistas de alto rendimiento y distan mucho de la media de los resultados de este estudio. Por otra parte, la fuerza y la flexibilidad son capacidades b sicas innatas de los seres humanos, donde el salto y la velocidad est $\mathrm{n}$ asociados a factores sociodemogr ficos como el g nero y la edad (Mathisen \& Pettersen, 2015, p.338), siendo la juventud la etapa donde se pueden desarrollar y obtener las mayores ganancias en estas capacidades $f$ sicas (Cornbleet \& Woolsty, 1996, p.852), similar a lo hallado en el presente estudio. Seg $\mathrm{n}$ lo anterior, los datos reportados por Ram rez et al. (2017, p.995) en estudiantes escolares colombianos, las medias de los resultados del test de salto son muy similares a los encontrados en este estudio, evidenci ndose poca diferencia entre los estudiantes escolares y universitarios, reflejando as un rea problem tica importante en los procesos de formaci $n$ y educaci $\mathrm{n}$ f sica, con poca estructuraci $\mathrm{n}$ de planes de entrenamiento espec ficos para potenciar las capacidades $f$ sicas, tanto a nivel escolar como universitario.

Tambi $n$ se encontr una correlaci $n$ directa entre la flexibilidad y la velocidad, e inversa con el salto, resultado que coincide con los hallazgos de Rodr guez \& And jar (2010, p.9), quienes mediante $t$ cnicas de estiramiento est tico y din mico, evaluaron el efecto agudo del rendimiento del test de velocidad de 30mts, encontrando que el estiramiento y la capacidad flexible del $\mathrm{m}$ sculo puede afectar de alguna forma la producci n de fuerza potencia. En el estudio de Garc a, Ruiz, More- 
no \& Latorre (2015, p.33) con jugadores de futbol americano, se midi la influencia de la flexibilidad de los isquiotibiales con el test de estiramiento pasivo en el salto y la velocidad, y se encontr que los jugadores que tuvieron mayor flexibilidad de los isquiotibiales, presentaron una mejor ejecuci $\mathrm{n}$ en los test de velocidad y de salto, resultados que difieren con los del presente estudio, debido a que la flexibilidad se correlacion directamente con la velocidad, pero no con el salto (Garc a et al., 2015, p.33).

Estas diferencias pueden estar asociadas a la ejecuci $n$ del test de Wells, donde hay mayor compromiso de m sculos de la cadena muscular posterior que pueden influir en el resultado del test, en tanto el test de estiramiento pasivo mide $\mathrm{m}$ s espec ficamente la flexibilidad de la musculatura isquiotibial ( $\mathrm{L}$ pez, Vaquero, Muyor \& Espejo, 2015, p.313). En un estudio realizado por Kirmizigil, Ozcaldiran \& Colakoglu (2014, p.1266), en el que se evaluaron tres $t$ cnicas de estiramiento y su influencia en el salto contra gravedad, se encontr que el estiramiento bal stico en la fase de calentamiento propici una mejor ejecuci n en altura del salto contra gravedad, comparado con el estiramiento est tico y la t cnica de estiramiento neuromuscular propioceptivo. Este resultado se asocia con lo hallado en el presente estudio, donde el salto tuvo una correlaci $n$ negativa con la flexibilidad, debido a que la t cnica de estiramiento utilizada en el test de Wells es est tica, buscando un $\mathrm{m}$ ximo alargamiento de la fibra muscular sin transferir la energ a el stica que se genera en los estiramientos bal sticos y que estimulan la fuerza explosiva (Kirmizigil et al., 2014, p.1266; Røksund et al., 2017, p.18).

En lo que respecta a la fuerza $m$ xima del soleo, tuvo una correlaci $\mathrm{n}$ inversa con la velocidad y directa con el salto, lo que estar a relacionado debido a que el soleo, por su anatom a y biomec nica, es un $\mathrm{m}$ sculo estabilizador y resistente, $\mathrm{m} \mathrm{s}$ que potente, siendo el primer mecanismo propulsor del salto, 
pero con poca activaci $\mathrm{n}$ en la velocidad (Lui, 2008, p.3246). El soleo tiene una mayor influencia en la aceleraci $n$ vertical del centro de gravedad y en el soporte articular en la fase de balanceo de la marcha (Liu, Anderson, Schwartz \& Delp, 2008, p.3246; Francis et al., 2013, p.268)

De la misma manera, esto se vincula con lo hallado en este estudio, donde la velocidad present una correlaci $\mathrm{n}$ inversa con el salto, pero se aleja bastante de lo reportado por Tejada \& Ram n (2013, p.7), donde los deportistas que fueron m s veloces, tuvieron mayor altura de salto.

\section{CONCLUSIONES}

En el estudio participaron 103 jugadores de ultimate frisbee de la ciudad de Medell $n$, donde las mujeres presentaron mayor flexibilidad que los hombres. Seg $\mathrm{n}$ los datos arrojados en el estudio, capacidades $f$ sicas como fuerza $m$ xima del soleo e isquiotibiales, velocidad y salto presentaron resultados $\mathrm{m}$ s elevados en los hombres que en las mujeres.

Existe una correlaci $n$ inversa de la flexibilidad de los isquiotibiales con respecto a la edad y el peso. A mayor flexibilidad de los isquiotibiales, mayor velocidad, pero menor saltabilidad (Aboodarda et al., 2015), y a mayor fuerza m xima del soleo, menor velocidad, pero mayor capacidad de salto. A mayor flexibilidad de los isquiotibiales, menor fuerza $\mathrm{m}$ xima de los mismos; en cambio, a mayor fuerza $m$ xima de los isquiotibiales, mejor salto y menor velocidad.

Siendo el ultimate frisbee un deporte reconocido por el Comit Ol mpico Internacional como postulante al programa ol mpico para los Juegos de Par s y Los ngeles, se abre un espectro de posibilidades para continuar contribuyendo a los procesos de investigaci $\mathrm{n}$ que permitan generar nuevos pasos para la formaci $n$ deportiva en todos sus niveles, y encontrar espacios 
para la actividad $\mathrm{f}$ sica, la diversi $\mathrm{n}$ sana y la inclusi $\mathrm{n}$ de la mujer de forma equitativa en el mundo deportivo.

\section{REFERENCIAS}

1. Aboodarda, S., Page, P., \& Behm, D. (2015). Eccentric and concentric jumping performance during augmented jumps with elastic resitance: A Meta-analysis. International Journal of Sports Physical Therapy, 10(6), 839-849.

2. Akinbola, M., Logerstedt, D., Hunter, A., \& Snyder, L. (2015). Ultimate frisbee injuries in a collegiate setting. International Journal of Sports Physical Therapy, 10(1), 75-84.

3. Arazi, H., Mohammadi, M., \& Asadi, A. (2014). Muscular adaptations to depth jump plyometric training: Comparison of sand vs. land surface. Interventional Medicine and Applied Science, 6(3), 125-130.

4. Arregui, J., \& Mart nez, V. (2001). Estado actual de las investigaciones sobre la flexibilidad en la adolescencia. Revista Internacional de Medicina y Ciencias de la Actividad F sica y el Deporte, 1(2), 127-135.

5. Asadi, A., \& Ram rez, R. (2016). Effects of cluster vs. traditional plyometric training sets on maximal-intensity exercise performance. Medicina, 52(1), 41-45.

6. Askling, C., Saartok, T., \& Thorstensson, A. (2006). Type of acute hamstring strain affects flexibility, strength, and time to return to preinjury level. British Journal of Sports Medicine, 40(1), 40-44.

7. Bandy, W., Irion, J., \& Briggler, M. (1997). The effect of time and frequency of static stretching on flexibility of the hamstring muscles. Physical Therapy, 77(10), 1090-1096.

8. Beedie, C. (2007). Placebo effects in competitive sports: qualitative data. Journal of Sports Science \& Medicine, 6(1), 21-28.

9. Chumanov, E., Heiderscheit, B., \& Thelen, D. (2011). Hamstring musculotendon dynamics during stance and swing phases of highspeed running. Medicine and Science in Sports and Exercise, 43(3), 525-532.

10. Cornbleet, S., \& Woolsty, N. (1996). Hamstring muscle length in school-aged children using the sit-and-reachtest and the inclinometer measure of hip joint angle. Physical Therapy, 76(8), 850-855.

11. Czaprowski, D., Leszczewska, J., Kolwicz, A., Pawlowska, P., Kedra, A., Janusz, P., \& Kotwicki, T. (2013). The comparison of the effects of three physiotherapy techniques on hamstring flexibility in children: a prospective, randomized, single-blind study. PLOS one, 8(8):e72026. 
12. Daneshjoo, A., Rahnama, N., Mokhtar, A., \& Yusof, A. (2013). Bilateral and unilateral asymmetries of isokinetic strength and flexibility in male young professional soccer players. Journal of Human Kinetics, 36(1), 45-53.

13. Domire, Z., \& Challis, J.(2015). Maximum height and minimum time vertical jumping. Journal of Biomechanics, 48(11), 2865-2870.

14. Francis, C., Lenz, A., Lenhart, R., \& Thelen, D. (2013). The modulation of forward propulsion, vertical support, and center of pressure by the plantarflexors during human walking. Gait Posture, 38(4), 993-997.

15. Gabbe, B., Finch, C., Bennell, K., \& Wajswelner, H. (2005). Risk factors for hamstring injuries in community level Australian football. British Journal of Sports Medicine, 39(2), 106-110.

16. Gambelli, C., Theisen, D., Willems, P., \& Schepens, B. (2015). Motor control of landing from a jump in simulated hypergravity. PLOS one, 10(10), e0141574.

17. Garc a, F., Ruiz, A., Moreno, R., \& Latorre, P. (2015). Impact of limited hamstring flexibility on vertical jump, kicking speed, sprint, and agility in young football players. Journal of Sports Science and Medicine, 33(12), 1293-1297.

18. Gonz lez, J., \& Gorostiaga, E. (2002). Fundamentos del entrenamiento de la fuerza. Aplicaci $n$ de alto rendimiento deportivo. Espa a: Inde.

19. Hammonds, A., Laudner, K., McCaw, S., \& McLoda, T. (2012). Acute lower extremity running kinematics after a hamstring stretch. Journal of Athletic Training, 47(1), 5-14.

20. Huerta, A., Chirosa, L., Guisado, R., \& C ceres, P. (2016). Acute effect of a complex training protocol of back squats on 30-m sprint times of elite male military athletes. Journal of Physical Therapy Science, 28(3), 752-756.

21. Irion, W., \& Briggler, M. (1997). The effect of time and frequency of static stretching on flexibility of the hamstring muscles. Physical Therapy. 77(10), 1090-1096.

22. Jenkins, N., Housh, T., Buckner, S., Bergstrom, H., Smith, C., Cochrane, K., et al. (2016). Four weeks of high- versus low-load resistance training to failure on the rate of torque development, electromechanical delay, and contractile twitch properties. Journal of Musculoskeletal and Neuronal Interactions, 1682), 135-144.

23. Johnson, E., \& Thomas, J. (2010). Effect of hamstring flexibility on hip and lumbar spine joint excursions during forward-reaching tasks in participants with and without low back pain. Archives of Physical Medicine and Rehabilitation, 91(7), 1140-1142.

24. Kirmizigil, B., Ozcaldiran, B., \& Colakoglu, M. (2014). Effects of three different stretching techniques on vertical jumping performance. Journal of Strength and Conditioning Research, 28(5), 1263-1271. 
25. L pez, P., Vaquero, R., Muyor, J., \& Espejo, L. (2015). Criterion-related validity of sit-and-reach test as a measure of hamstring extensibility in older women. Nutrici n Hospitalaria, 32(1), 312-317.

26. Liu, M., Anderson, F., Schwartz, M., \& Delp, S. (2008). Muscle contributions to support and progression over a range of walking speeds. Journal of Biomechanics, 41(15), 3243-3252.

27. Malliaropoulos, N., Kakoura, L., Tsitas, K., Christodoulou, D., Siozos, D., Malliaras, P., \& Maffuli, N. (2015). Active knee range of motion assessment in elite track and field athletes: normative values. Muscles, Ligaments and Tendons Journal, 5(3), 203-207.

28. Mathisen, G., \& Pettersen, S. (2015). Anthropometric factors related to sprint and agility performance in young male soccer players. Open Access Journal of Sports Medicine, 6,337-342.

29. Muehlbauer, T., Gollhofer, A., \& Granacher, U. (2015). Associations between measures of balance and lower-extremity muscle strength/ power in healthy individuals across the lifespan: A systematic review and meta-analysis. Sports Medicine, 45(12), 1671-1692.

30. Nishikawa, Y., Aizawa, J., Takahashi, N., Hosomi, N., Maruyama, H., \& Kimura, H. (2015). Immediate effect of passive and active stretching on hamstrings flexibility: a single-blinded randomized control trial. Journal of Physical Therapy Science, 27(10), 3167-3170.

31. Ram rez, R., Correa, J., Lobelo, F., Cadore, E., Alonso, A., \& Izquierdo, M. (2017). Vertical jump and leg power normative data for colombian schoolchildren aged 9-17.9 years: The FUPRECOL study. Journal of Strength and Conditioning Research, 31(4), 990-998.

32. Reynolds, K., \& Halsmer, S. (2006). Injuries from ultimate frisbee. Wisconsin Medical Journal, 105(6), 46-49.

33. Rodr guez, F., \& And jar, P. (2010). Acute effect of stretching on sprint in honour division soccer players. International Journal of Sport Science, 6, 1-12.

34. Rogan, S., W st, D., Schwitter, T., \& Schmidtbleicher, D. (2015). Static stretching of the hamstring muscle for injury prevention in football codes. Asian Journal of Sports Medicine, 4(1), 1-9.

35. Røksund, O. D., Kristoffersen, M., Bogen, B. E., Wisnes, A., Engeseth, M. S., Nilsen, A. K., ... \& Gundersen, H. (2017). Higher drop in speed during a repeated sprint test in soccer players reporting former hamstring strain injury. Frontiers in Physiology, 8(25), 1-8.

36. Sabido, R., Hern ndez, J., Botella, J., \& Moya, M. (2016). Effects of 4-Week training intervention with unknown loads on power output performance and throwing velocity in junior team handball players. PLOS one, 11(6), e0157648. 
37. Smith, M., Greenlees, I., \& Manley A. (2009). Influence of order effects and mode of judgement on assessments of ability in sport. Journal of Sports Sciences, 27(7), 745-752.

38. Tejada, C., \& Ram n, G. (2013). Correlaci n entre la potencia en miembros inferiores (altura de despegue del salto) medida con protocolo de Bosco y la velocidad frecuencial (medida con el test de 30 y 60 metros planos) de la selecci n Colombia femenina y masculina de ultimate frisbee. VIREF Revista de Educaci n F sica, 2(1), 147-162.

39. Valle, X., Hamilton, B., Rodas, G., Malliaras, P., Malliaropoulos, N., et al. (2015). Hamstring muscle injuries, a rehabilitation protocol purpose. Asian Journal of Sports Medicine, 6(4), e25411.

40. Wong, J., Bobbert, M., Soest, A., Gribble, P., \& Kistemaker, D. (2016). Optimizing the distribution of leg muscles for vertical jumping. PLOS one, 11, 1-15. 